\title{
COVID-19 y metabolismo: una mirada más allá del sistema respiratorio y de la enfermedad trombótica
}

\section{COVID-19 and metabolism: a look beyond the respiratory system and thrombotic disease}

\author{
Óscar L. Medina-Espitia1 , Fernán Mendoza-Beltrán² ${ }^{2}$, Ana M. Anaya-Almanza³ y \\ Óscar A. Molano-Salazar ${ }^{3}$ \\ ${ }^{1}$ Servicio de Endocrinología; ${ }^{2}$ Departamento de Cardiología Clínica y Medicina Interna; ${ }^{3}$ Médicos rotantes, Fundación Clínica Shaio. Fundación \\ Clínica Shaio, Bogotá, Colombia
}

\section{Resumen}

La COVID-19 es una enfermedad infecciosa causada por el coronavirus del síndrome respiratorio agudo severo 2 (SARSCoV-2) que tiene importantes manifestaciones sobre el sistema cardiovascular y respiratorio. Esta enfermedad, descrita en los primeros días de diciembre de 2019 en la ciudad de Wuhan, capital de la provincia de Hubei, en China, tuvo una rápida expansión mundial y fue declarada por la Organización Mundial de la Salud como pandemia el 11 de marzo de 2020 . Tiene diferentes presentaciones clínicas, como neumonía, hipoxemia, falla renal, falla multisistémica, compromiso endotelial que lleva a lesiones trombóticas venosas y arteriales, y problemas cardiacos como insuficiencia cardiaca, miocarditis, arritmias e infarto de miocardio de los tipos 1 y 2. La mayoría de las personas presentan una enfermedad leve o no complicada (80\%), y otras (20\%) pueden desarrollar un cuadro grave con neumonía, síndrome de dificultad respiratoria, choque cardiogénico, trombosis y tormenta de citocinas (el $15 \%$ se manejan con oxigenoterapia y el $5 \%$ ameritan tratamiento en la unidad de cuidados intensivos). La mortalidad está relacionada con la edad y con la comorbilidad (hipertensión arterial, diabetes mellitus, enfermedades respiratorias, enfermedades cardiacas en general, enfermedades renales y obesidad). El objetivo de esta revisión narrativa es describir las alteraciones metabólicas y la relación de la diabetes mellitus, la obesidad, el síndrome metabólico y la dislipidemia con la morbilidad y la mortalidad vinculadas a la COVID-19.

Palabras clave: Coronavirus. SARS-CoV-2. Diabetes mellitus. Obesidad. Dislipidemia. Enzima convertidora de la angiotensina 2.

\section{Abstract}

COVID-19 is an infectious disease caused by the severe acute respiratory syndrome coronavirus 2 (SARS-CoV-2) and that has important manifestations on the cardiovascular system and respiratory. This disease described in the first days of December 2019 in the city of Wuhan, capital of the province of Hubei, China, with a rapid global expansion and declared by the World Health Organization as a pandemic on March 11, 2020, has different clinical presentations such as pneumonia, hypoxemia, kidney failure, multisystem failure, endothelial involvement leading to venous and arterial thrombotic lesions, and heart problems such as heart failure, myocarditis, arrhythmias, and type 1 and 2 myocardial infarction. Most people have a mild or uncomplicated disease (80\%), and others (20\%) may develop a severe case with pneumonia, respiratory distress syndrome,

\section{Correspondencia:}

*Fernán Mendoza-Beltrán

E-mail: fernan.mendoza@shaio.org
Disponible en internet: 18-10-2021 Rev Colomb Cardiol. 2021;28(4):366-373 www.rccardiologia.com 0120-5633 / C 2021 Sociedad Colombiana de Cardiología y Cirugía Cardiovascular. Publicado por Permanyer. Este es un artículo open access bajo la licencia CC BY-NC-ND (http://creativecommons.org/licenses/by-nc-nd/4.0/). 
cardiogenic shock, thrombosis, and cytokine storm (15\% are managed with oxygen therapy and 5\% merit treatment in the intensive care unit). Mortality is related to age and comorbidity (high blood pressure, diabetes mellitus, respiratory diseases, heart diseases in general, kidney diseases, and obesity). The objective of this narrative review is to describe the metabolic alterations and the relationship between diabetes mellitus, obesity, metabolic syndrome, and dyslipidemia with the morbidity and mortality associated with COVID-19 infection.

Key words: Coronavirus. SARS-CoV-2. Diabetes mellitus. Obesity. Dyslipidemia. Angiotensin-converting enzyme 2.

\section{Introducción}

El coronavirus es un virus ARN con un tamaño de 27-32 kilobases, de la familia Coronaviridae, que ha sido descrito como un agente zoonótico que afecta el tejido entérico y respiratorio de los animales infectados. En los humanos produce alteraciones respiratorias, la mayoría de las veces autolimitadas, y cada año es causa de un $15-30 \%$ de las infecciones de vías respiratorias altas ${ }^{1}$. Los coronavirus se pueden diferenciar en cuatro géneros, alfa, beta, delta y gamma; de ellos, hasta el momento se sabe que los alfa y los beta son los que infectan a los humanos.

El coronavirus asociado al síndrome respiratorio agudo grave (SARS-CoV) fue descubierto en 2002 en Guangdong (China) como agente etiológico de la enfermedad a la debe su nombre. Posteriormente, en 2012, se describió el virus relacionado con el síndrome respiratorio de Oriente Medio (MERS-CoV), el cual presentó una tasa de mortalidad del $50 \%$ hasta que la epidemia se controló en 2014. En 2019 apareció el SARS-CoV-2, un agente que originó el síndrome respiratorio agudo grave y que se expandió de Wuhan (China) al resto del mundo, declarándose como emergencia de salud pública el 30 de enero de 2020 y 3 meses después, el 11 de marzo, como pandemia mundial por la Organización Mundial de la Salud'. En el momento de escribir esta revisión, Colombia reportaba ya $1,686,131$ infectados y 44,187 fallecidos, con un número reproductivo efectivo que pasó de 2.28 al principio de la pandemia a 1.11 y una tasa de letalidad que pasó del $5 \%$ al $3.18 \%$.

Justificado por las estadísticas mundiales y de Colombia, donde la mortalidad por la infección está relacionada con la comorbilidad de los pacientes (hipertensión arterial $23 \%$, diabetes mellitus $23 \%$, enfermedades respiratorias $15 \%$, enfermedades cardiovasculares $8 \%$ y obesidad $8 \%$ ), el objetivo de esta revisión es describir las alteraciones metabólicas y la relación entre la diabetes mellitus, la obesidad, el síndrome metabólico y la dislipidemia con la morbilidad y la mortalidad vinculadas a la COVID-19.

\section{Obesidad y adiposidad como predictores de desenlaces de la COVID-19}

La obesidad se ha asociado a un curso clínico más grave de las infecciones virales, entre ellas la influenza asiática, la influenza de Hong Kong y la pandemia por virus H1N1 de 1957, 1968 y 2009, respectivamente².

Para comprender la asociación de la COVID-19 y la obesidad es necesario recordar los mecanismos de entrada del SARS-CoV-2 al organismo, que dependen de la fusión de la membrana viral y de la membrana celular del huésped, mediada por trímeros de la proteína $\mathrm{S}$ del virus (S1 y S2) que se unen al heterodímero de la enzima convertidora de angiotensina 2 (ECA2) ${ }^{2,3}$, dando como resultado la liberación de ARN viral al citoplasma ${ }^{4}$. Los mayores grados de expresión de la ECA2 se realizan en el intestino delgado, los testículos, los riñones, el corazón, la tiroides, el tejido adiposo, el pulmón, el colon, el hígado, la vejiga y las glándulas adrenales, dejando por último los tejidos con menor expresión de la enzima, entre los que se encuentran el bazo, la médula ósea, el cerebro, el músculo y los vasos sanguíneos. Esto explica la afectación multisistémica de la enfermedad ${ }^{5}$.

Posterior a la integración del virus en la célula huésped, la infección resulta en un aumento de la apoptosis celular y un reclutamiento de células inflamatorias, causando la apoptosis de células CD3, CD4 y CD8, lo que desencadena la tormenta de citocinas, caracterizada por la elevación de las interleucinas (IL) 6, 7 y 2, el factor de necrosis tumoral alfa (TNF- $\alpha$ ), el ligando CXC de quimiocinas, la proteína quimioatrayente de monocitos 1 (MCP-1) y otras moléculas inflamatorias. En presencia de obesidad, los adipocitos hipertróficos producen gran cantidad de citocinas, como IL-8 e IL-6, inhibidor del activador de plasminógeno 1, leptina y MCP-1/CCL2, lo que lleva a un aumento en el reclutamiento de macrófagos, especialmente M1, que a su vez producen moléculas inflamatorias similares a las descritas previamente (IL-1 $\beta$, IL-6, IL-8, TNF $\alpha$ y MCP-1) ${ }^{2}$.

Todo lo anterior lleva a una alteración de la inmunidad innata en estos pacientes, pero la inmunidad 
Tabla 1. Eventos desencadenados en el síndrome metabólico, la diabetes mellitus y la COVID-19

\begin{tabular}{|c|c|c|}
\hline Patología/eventos & $\begin{array}{l}\text { Síndrome } \\
\text { metabólico y } \\
\text { diabetes mellitus }\end{array}$ & COVID-19 \\
\hline Inflamatorios & $\begin{array}{l}\text { Inflamación } \\
\text { crónica }\end{array}$ & $\begin{array}{l}\text { Tormenta de } \\
\text { citocinas }\end{array}$ \\
\hline Cardiometabólicos & $\begin{array}{l}\text { Hipertensión, } \\
\text { hiperglucemia, } \\
\text { disfunción } \\
\text { endotelial, } \\
\text { alteraciones } \\
\text { metabólicas }\end{array}$ & $\begin{array}{l}\text { Daño multiorgánico, } \\
\text { hipercoagulación, } \\
\text { hipoxia muscular, } \\
\text { alteraciones } \\
\text { electrolíticas }\end{array}$ \\
\hline Renales & $\begin{array}{l}\text { Lesión renal } \\
\text { crónica }\end{array}$ & $\begin{array}{l}\text { Infección directa, } \\
\text { hipoxia, lesión renal } \\
\text { aguda }\end{array}$ \\
\hline Pulmonares & $\begin{array}{l}\text { Fibrosis, alteración } \\
\text { del surfactante, } \\
\text { alteración del } \\
\text { movimiento } \\
\text { diafragmático }\end{array}$ & $\begin{array}{l}\text { Daño de la barrera } \\
\text { alveolar, neumonía, } \\
\text { edema, alteración } \\
\text { en la difusión de } \\
\text { oxígeno, alteración } \\
\text { de la composición y } \\
\text { la cantidad de } \\
\text { surfactante }\end{array}$ \\
\hline Nerviosos & $\begin{array}{l}\text { Alteración } \\
\text { cognitiva de } \\
\text { origen vascular, } \\
\text { demencia, ataque } \\
\text { cerebrovascular, } \\
\text { alteración de la } \\
\text { glucemia, } \\
\text { convulsiones }\end{array}$ & $\begin{array}{l}\text { Ataque } \\
\text { cerebrovascular, } \\
\text { convulsiones, } \\
\text { alteración cognitiva }\end{array}$ \\
\hline
\end{tabular}

Adaptada de Ayres?.

adaptativa también se encuentra afectada, con disminución de las células $T$ reguladoras y tendencia hacia líneas proinflamatorias Th1, Th17 y Th222,6. El efecto final es el solapamiento de un aumento de citocinas desencadenado en la infección por SARS-Cov-2, sobre la base proinflamatoria de estos pacientes.

Los eventos previos en los pacientes con síndrome metabólico, obesidad y diabetes mellitus empeoran en presencia de infección por SARS-CoV-2; algunos de ellos se describen en la tabla $1^{7}$.

Posterior a la infección por SARS- CoV-2 se han observado complicaciones a largo plazo, entre las que se encuentran alteración en la sensibilidad a la insulina, daño en los islotes pancreáticos con reducción en la secreción de insulina, debilidad y atrofia muscular con cambios en la capacidad para realizar ejercicio, cambios en la composición corporal con aumento de la masa grasa, y elevación de los triglicéridos y de los ácidos grasos circulantes; todas ellas podrían conducir finalmente a un aumento del riesgo de eventos cardiovasculares futuros ${ }^{7}$.

Se ha asociado la presencia de obesidad con peores desenlaces clínicos de la COVID-19. En un estudio realizado en Nueva York con más de 4000 pacientes, el principal predictor para la hospitalización fue la edad mayor de 75 años (odds ratio [OR]: 66.8; intervalo de confianza del 95\% [IC95\%] 44.7-102.6), seguida del índice de masa corporal (IMC) > 40 kg/m² (OR: 6.2; IC95\%: 4.2-9.3 $)^{8}$. El estudio del Bronx, que incluyo 200 participantes, concluyo que el IMC $>35 \mathrm{~kg} / \mathrm{m}^{2}$ es una variable independiente asociada a mortalidad en pacientes hospitalizados (OR: 3,78; IC95\%: 1.45-9.83; $p<0.006$ ).

En un metaanálisis que incluyó 14 estudios se reportó que un IMC > 25 kg/m² (OR: 3.68; IC95\%: 1.54-8.83; $\mathrm{p}=0.003$ ), la presencia de comorbilidad grave (OR: 1.84; IC95\%: 1.61-2.09; $p<0.00001)$, necesitar soporte ventilatorio (OR: 6.98; IC95\%: 5.37-9.07; $p<0.00001$ ) y desarrollar enfermedad grave (OR: 2.03; IC95\%: 1.752.36; $p$ < 0.00001) se asociaron a mayor mortalidad en los pacientes con infección por SARS-CoV-2 ${ }^{10}$.

Por otro lado, la distribución de la grasa corporal también está relacionada con efectos no deseables. En un estudio realizado en Alemania se reportó que cada centímetro adicional de circunferencia abdominal aumenta 1.25 veces la probabilidad de ventilación mecánica $(p=0.008)$ y 1.13 veces la probabilidad de ingreso en la unidad de cuidados intensivos $(p=0.009)^{11}$.

\section{Relación entre diabetes mellitus tipo 2 y COVID-19}

La diabetes mellitus tipo 2 se ha considerado un factor de riesgo para adquirir la infección por coronavirus. Históricamente, la hipertensión arterial y la diabetes han sido identificadas como la comorbilidad más común para otras infecciones por coronavirus, tales como el SARS-CoV y el MERS-CoV'12.

Dependiendo de la región y de las series analizadas, la prevalencia y el riesgo de complicaciones cambian en forma importante. La prevalencia media de diabetes en pacientes diagnosticados de infección por SARSCoV-2 es del $7-10 \%$, y llega hasta el $35 \%{ }^{13}$ (Tabla 2$)^{14}$.

\section{¿Aumenta la diabetes mellitus tipo 2 el riesgo de complicaciones y de muerte por COVID-19?}

De acuerdo con diferentes reportes, los pacientes con diabetes mellitus tipo 2 y síndrome metabólico tienen 
Tabla 2. Desenlaces en personas con COVID-19 de acuerdo con el control glucémico

\begin{tabular}{|c|c|c|c|c|c|c|}
\hline Autores & Tipo de estudio & Población & $\begin{array}{l}\text { Prevalencia } \\
\text { de diabetes }\end{array}$ & Parámetros & Desenlace & $\begin{array}{l}\text { HR y OR } \\
\text { ajustadas }\end{array}$ \\
\hline Williamson, et al. & Estudio de cohorte & $17,425,445$ & $10 \%$ & $\mathrm{HbA} 1 \mathrm{c} \geq 7.5 \%$ & Mortalidad & $2.36(2.18-2.56)$ \\
\hline Holman, et al. & Estudio de cohorte & $2,889,210$ & $100 \%$ DM2 & $\mathrm{HbA} 1 \mathrm{c} \geq 7,5 \%$ & Mortalidad & $1.62(1.48-1.79)$ \\
\hline Li, et al. & Estudio retrospectivo & 269 & $19 \%$ & $\begin{array}{l}\text { Hiperglucemia durante } \\
\text { hospitalización }\end{array}$ & Mortalidad & $1.77(1.11-2.84)$ \\
\hline Chen, et al. & Estudio retrospectivo & 904 & $15 \%$ & Hiperglucemia & Mortalidad & $1.08(1.01-1.16)$ \\
\hline Zhu, et al. & Estudio retrospectivo & 818 & $100 \%$ & $\begin{array}{l}\text { Glucemia media durante } \\
\text { estancia hospitalaria } 6-4 \\
\text { mmol// (RIO: } 5.2-7.5)\end{array}$ & SDRA & $0.41(0.25-0.66)$ \\
\hline Zhu, et al. & Estudio retrospectivo & 818 & $100 \%$ & $\begin{array}{l}\text { Glucemia media durante } \\
\text { estancia hospitalaria } 6-4 \\
\mathrm{mmol} / \mathrm{l}(\mathrm{RIO}: 5.2-7.5)\end{array}$ & $\begin{array}{l}\text { Lesión } \\
\text { miocárdica }\end{array}$ & $0.21(0.07-0.59)$ \\
\hline
\end{tabular}

DM2: diabetes mellitus tipo 2; HbA1c: hemoglobina glucosilada; HR: hazard ratio; OR: odds ratio; RIQ: rango interquartílico; SDRA: síndrome de dificultad respiratoria aguda.

Adaptada de Apicella, et al. ${ }^{14}$

mayor riesgo de muerte, siendo estas afecciones predictoras independientes de morbilidad y mortalidad ${ }^{12}$.

En su metaanálisis de mortalidad y morbilidad, Kumar, et al. ${ }^{15}$ incluyeron 16,003 pacientes con diagnóstico de COVID-19 de 33 estudios. La prevalencia de diabetes mellitus tipo 2 en los pacientes con COVID-19 fue del 9.8\% (IC95\%: 8.7-10.9). La diabetes se asoció a mortalidad con una OR de 1.9 (IC95\%: 1.37-2.64; $p<0.01$ ), e igualmente con la gravedad con una OR de 2.75 (IC95\%: 2.09-3.62; $p<0.01$ ) y un aumento en el desenlace combinado de mortalidad o gravedad con una OR de 2.16 (IC95\%: 1.74-2.68; $p<0.01)^{15}$.

Mantovani, et al. ${ }^{16}$, en su metaanálisis de gravedad y mortalidad intrahospitalaria, incluyeron 78,874 pacientes hospitalizados tomados de 83 estudios con diagnóstico confirmado de COVID-19. La prevalencia en pacientes con diabetes mellitus tipo 2 fue del $14.3 \%$ (IC95\%: 12.6-16.0). Llama la atención que la prevalecia de diabetes mellitus tipo 2 fue mayor en los no asiáti$\cos (23.3$ vs $11.0 \%)$ y en los mayores de 60 años (23.3 vs $8,79 \%)$. La preexistencia de diabetes se asoció a dos veces más riesgo de tener COVID-19 grave (OR: 2.1; IC95\%: 1.71-2.5; I2 = 41.5\%) y tres veces más riesgo de morir (OR: 2.68; IC95\%: 2.0-3.4; I2 = 46\%).

Huang, et al. ${ }^{17}$, en un metaanálisis y metarregresión, evaluaron la diabetes mellitus tipo 2 en relación con el aumento de la mortalidad y la gravedad de la COVID-19 en presencia de neumonía. Incluyeron 6452 pacientes de 30 estudios. El metaanálisis mostró que la diabetes se asoció a un pobre desenlace compuesto (riesgo relativo [RR]: 2.38; IC95\%: 1.88-3.03; $p<0.001$ ), un aumento en la mortalidad (RR: 2.12; IC95\%: 1.44-3.11; $\mathrm{p}<0.001)$ y mayor gravedad (RR: 2.45; IC95\%: 1.79$3.35 ; p<0.001)$, y significativamente aumentó la probabilidad de síndrome de dificultad respiratoria aguda (RR: 4.64; IC95\%: 1.44-3.11; $p<0.001$ ) y de progresión de la enfermedad (RR: 3.31; IC95\%: 1.08-10.14; $p<0.001$ ). La metarregresión mostró que la asociación con el pobre desenlace compuesto fue influenciada por la edad $(p=0.003)$ y la hipertensión $(p<0.001)^{16}(\text { Tabla } 3)^{17}$.

\section{Factores metabólicos de riesgo para desenlaces adversos}

La informacion más importante fue generada por el estudio CORONADO ${ }^{18}$, un estudio multicéntrico obsevacional realizado en pacientes con diabetes hospitalizados por COVID-19 en 53 centros. El desenlace primario combinado fue intubación orotraqueal o muerte en los 7 días luego de la hospitalización, con la intención de determinar las variables clínicas y biológicas relacionadas con el desenlace. El análisis final fue aplicado en 1314 participantes, de los que el $88 \%$ tenían diabetes mellitus tipo 2, el $64 \%$ eran hombres, la edad media era de 69.8 años y el IMC medio era de $28.4 \mathrm{~kg} / \mathrm{m}^{2}$. El desenlace primario ocurrió en el 29\% (IC95\%: 26.6-31.5; $p<0.005$ ) y el $10.6 \%$ de los participantes fallecieron. En el análisis multivariado, las características previas al ingreso asociadas de manera significativa con el desenlace primario fueron el sexo masculino, el IMC y el tratamiento previo con bloquedores del sistema renina-angiotensina-aldosterona. No influyeron la edad, el 
Rev Colomb Cardiol. 2021;28(4)

Tabla 3. Desenlaces en personas con COVID-19 y diabetes preexistente

\begin{tabular}{|l|l|c|c|l|c|}
\hline Autores & Tipo de estudio & Población & Prevalencia de diabetes & Desenlace & HR y OR ajustadas \\
\hline Kumar, et al. & Metaanálisis & 16,003 & $9.8 \%$ & Enfermedad grave & $2.75(2.09-3.62)$ \\
\hline Kumar, et al. & Metaanálisis & 16,003 & $9.8 \%$ & Mortalidad & $1.90(1.37-2.64)$ \\
\hline Li, et al. & Metaanálisis & 1525 & $9.7 \%$ & Admisiones a UCI & $2.21(0.88-5.57)$ \\
\hline Fadini, et al. & Metaanálisis & 1687 & No datos & Enfermedad grave & $2.26(0.98-4.82)$ \\
\hline Fadini, et al. & Metaanálisis & 355 & $35.5 \%$ & Mortalidad & 1.75 \\
\hline Roncon, et al. & Metaanálisis & 1382 & No datos & Admisiones a UCI & $2.79(1.85-4.22)$ \\
\hline Roncon, et al. & Metaanálisis & 471 & No datos & Mortalidad & 3.21 (1.82-5.64)
\end{tabular}

HR: hazard ratio; OR: odds ratio; UCl: unidad de cuidados intensivos. Adaptada de Apicella, et al. ${ }^{14}$

tipo de diabetes, la hemoglobina glucosilada (HbA1c), las complicaciones previas relacionadas con la diabetes ni el tipo de terapia previa para esta. En el análisis multivariado con covariables previo a la admisión, solo el IMC permanecía positivamente asociado con el desenlace primario (OR: 1.28; IC95\%: 1.10-3.35; $p<0.005$ ). El riesgo de muerte al séptimo día fue evaluado en variables independientes, encontrando asociación con la edad (OR: 2.48; IC95\%: 1.74-3.53; $p<0.005$ ), el tratamiento para apnea del sueño (OR: 2.80; IC95\%: 1.46- 5.38; $p<0.005$ ) y las complicaciones microvasculares (OR: 2.14; IC95\%: 1.16-3.94; $p<0.005$ ) y macrovasculares (OR: 2.54; IC95\%: 1.44-4.50; $p<0.005)^{18}$.

Los valores de HbA1c no influyeron en los desenlaces en el estudio CORONADO, pero en otros sí se ha encontrado asociación. Wang, et al. ${ }^{19}$ realizaron un estudio retrospectivo de 132 pacientes con COVID-19 asignados a tres grupos según la cifra de $\mathrm{HbA1c}$, con mediciones simultáneas de saturación arterial de oxígeno y de marcadores de inflamación sistémica. El grupo A tenía una $\mathrm{HbA1c}$ media del $5.7 \%$, el grupo B del $6.4 \%$ y el grupo $C$ del $7.5 \%$. Los grupos $B$ y $C$ tenían valores más altos de proteína $C$ reactiva $(P C R)$, velocidad de sedimentación globular (VSG), fibrinógeno, IL-6 y feritina sérica $(p<0.05)$. El análisis de correlación mostró una relación lineal negativa entre la saturación arterial de oxígeno y los valores de $\mathrm{HbA} 1 \mathrm{c}$ $(p=0.01)$, y una relación lineal positiva con los valores de ferritina, PCR, fibrinógeno y VSG $(p<0.05)^{19}$.

\section{Relación endocrinológica directa}

La ECA2 tiene como función la transformación de angiotensina I en angiotensina 1-9 y de angiotensina II en angiotensina 1-7, actuando como el punto regulatorio clave para el sistema de la angiotensina. La combinación de una actividad de la ECA-1 incrementada y de la ECA-2 inhibida eleva la angiotensina II intacta, favorece una respuesta proinflamatoria y estimula la secreción de aldosterona. Este efecto no solo aumenta la presión arterial y modifica los niveles de potasio, sino que también incrementa la permeabilidad vascular y aumenta el riesgo de síndrome de dificultad respiratoria agudo. La angiotensina 1-7 tiene el efecto contrario, con potenciales efectos antiinflamatorios y antifibróticos. Se ha postulado que los individuos con formas más graves de COVID-19 tienen un disbalance en la activación de estas vías, con un incremento en la activación de la angiotensina II que actúa sobre el receptor de la angiotensina 1 (AT1), lo cual ocurre en presencia de diabetes mellitus tipo 2, hipertensión y estados de resistencia a la insulina ${ }^{12}$.

Los mecanismos específicos que explican la asociación entre diabetes mellitus tipo 2 y SARS-CoV-2 podrían estar relacionados con:

- Los valores elevados de glucosa pueden aumentar directamente las concentraciones de glucosa en las secreciones de la vía aerea. Los estudios in vitro muestran que la exposición a valores de glucosa elevados en el epitelio pulmonar aumenta de manera significativa la infección por el virus de la influenza y su replicación. En general se acepta que los pacientes con diabetes mellitus tipo 2 cursan con mayores cargas virales o desarrollan formas más graves en caso de infección por virus respiratorios ${ }^{6}$.

- La hiperglucemia crónica afecta la función pulmomar. En modelos animales se han documentado un 


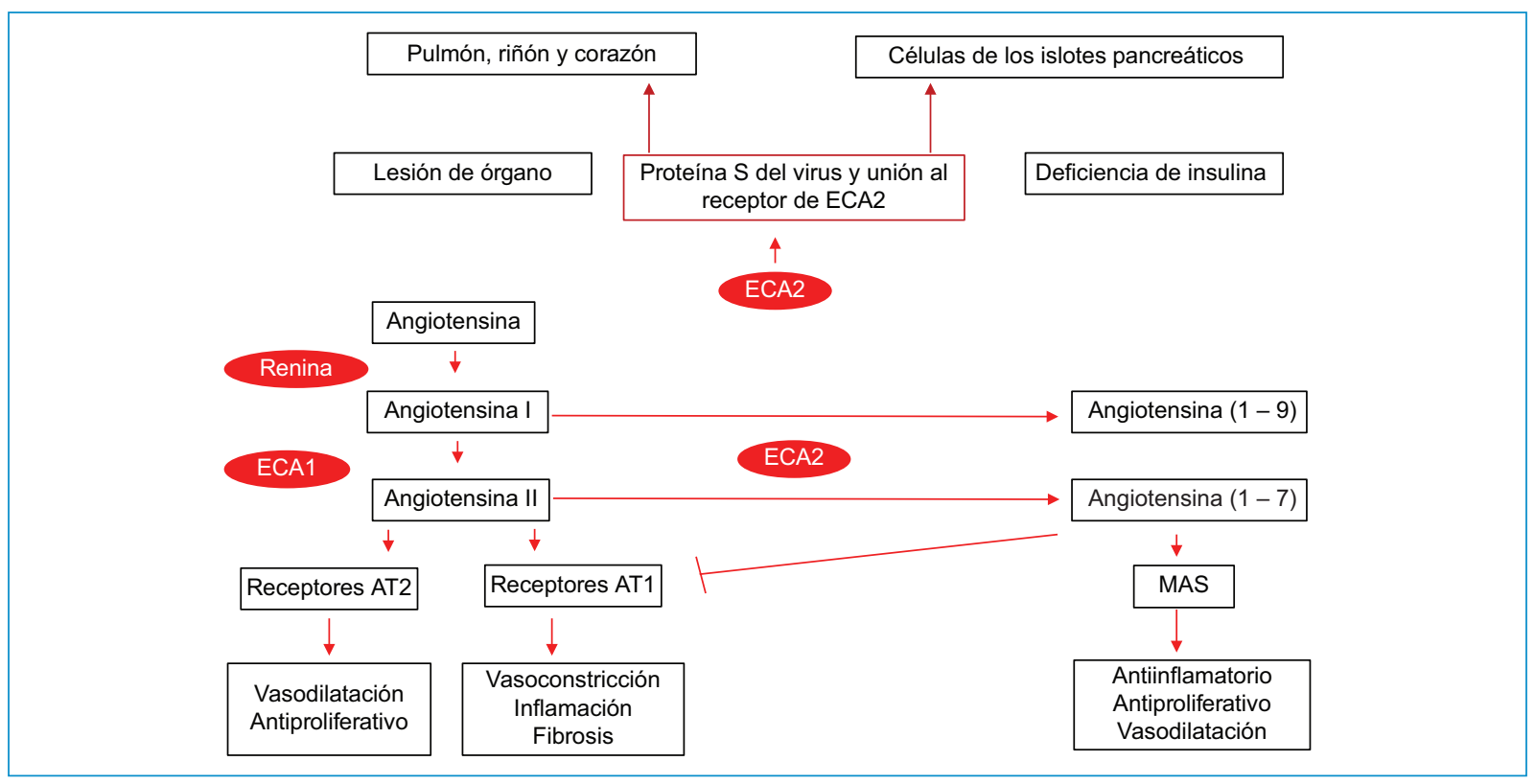

Figura 1. Relación entre el SARS-CoV-2 y SRAA. AT1: receptor tipo 1 de la angiotensina II; AT2: receptor tipo 2 de la angiotensina II; ECA1: enzima convertidora de angiotensina 1; ECA2: enzima convertidora de angiotensina 2; MAS: receptor acoplado a la proteína G; SRAA: sistema renina angiotensina aldosterona.

aumento de la permeabilidad de la membrana alveolocapilar y un colapso del epitelio alveolar ${ }^{6}$.

- El páncreas endocrino expresa el receptor ECA2, y la unión del SARS-CoV-2 al receptor le permite entrar a las células de los islotes pancreaticos y causar disfunción aguda de las celulas beta, lo cual puede generar hiperglucemia aguda o modificar el control glucémico previo ${ }^{12}$.

- Los pacientes con diabetes mellitus tipo 2 presentan mayor riesgo de infección, principalmente por defectos en la inmunidad innata que afectan a la fagocitosis, la quimiotaxis de neutrófilos y la inmunidad celular $^{13}$

- En humanos se ha demostrado que la hiperglucemia aguda regula al alta la expresión del receptor ECA2, facilitando que el virus penetre en la célula. La presencia de ECA2 inducida en otros tejidos, incluyendo pulmón, hígado y corazón, podría favorecer la inclusión del virus en las células, con un aumento en la probabilidad de falla multiorgánica ${ }^{13}$.

- Por el contrario, en la hiperglucemia crónica se observa una reducción en la expresión de ECA2, con cambio en la relación angiotensina II/angiotensiona 1-7, como ya se explicó. Este fenómeno favorecería el evento inflamatorio generado por el virus, agravando la inflamación crónica de bajo grado preexistente en los pacientes con diabetes mellitus tipo 2 , obesidad y síndrome metabólico, y empeorando el síndrome de liberación de citocinas con una respuesta inflamatoria aumentada y no controlada ${ }^{13,20}$.

- Incremento en los valores de plasmina y de furina, que igualmente están involucradas en la entrada del virus a la célula ${ }^{21}$.

La ECA2 convierte la angiotensina I y la angiotensina II en angiotensina 1-9 y angiotensina 1-7, respectivamente. La ECA2 se expresa en el pulmón, el riñón, el corazón y el páncreas, y actúa como facilitador para la entrada del SARS-CoV-2 en la célula. Estas facilidades en la expresión viral durante la entrada del virus a la célula del huésped causan lesiones orgánicas y deficiencia de insulina, contribuyendo así a la hiperglucemia. El incremento de la ECA2 podría convertir la angiotensina II en angiotensina 1-7, que actúa sobre el receptor Mas1 para desencadenar efectos antiinflamatorios e inhibe el receptor AT1, favoreciendo la vasodilatación. Sin embargo, al menos en los usuarios de fármacos inhibidores de la ECA (IECA), los valores de angiotensina II serán bajos y el beneficio neto de la regulación positiva de ECA2 es incierto. La infección por SARS-CoV-2 regula a la baja la expresión de ECA2, lo que disminuye la angiotensina 1-7, reduciendo sus efectos antiinflamatorios y potencialmente empeorando la vulnerabilidad del órgano a la infección (Fig. 1). 
Las diferentes sociedades científicas del mundo, a pesar de todo lo mencionado, recomiendan que los pacientes que vienen utilizando IECA y antagonistas de los receptores de la angiotensina II (ARA II) para la hipertensión arterial o para la insuficiencia cardiaca no los suspendan, ya que pueden generar más daño que beneficio. En el congreso de la Sociedad Europea de Cardiología de 2020 se presentó el estudio BRACE-CORONA, que refuerza que no hay justificación para suspender los IECA y los ARA II en los pacientes que los vienen tomando y presentan COVID-19.

\section{Dislipidemia y COVID 19}

Aparte de promover el transporte reverso del colesterol desde la periferia hasta el hígado, la lipoproteína de alta densidad (HDL) tiene otras propiedades, como la neutralización del lipopolisacárido y el ácido lipoteicoico presentes en agentes patógenos, y propiedades antiinflamatorias, antitrombóticas, protectoras antioxidantes y antiapoptosis, y de protección del endotelio ${ }^{22,23}$.

La interacción de los receptores Toll like de macrófagos y células $T$ con las moléculas de apolipoproteína A-I y M (ApoE y ApoC-III en menor medida) de HDL participan en la respuesta a estados como sepsis, neumonía y primoinfección por el virus de la inmunodeficiencia humana $(\mathrm{VIH})^{24}$ considerándose parte importante de los mecanismos moduladores del sistema inmunitario 22 .

El estado inflamatorio generado por la respuesta a la infección por SARS-CoV-2 induce respuestas que son modificadas por la HDL mediante las ApoA-I y Apo-E, por su actividad inmunomoduladora y antioxidante ${ }^{22,23}$. Por medio de las ApoE y ApoC-III presentes en la HDL se genera una disminución de la actividad de la lipoproteína lipasa. La señalización de radicales libres, la cual está elevada en las células huésped con una infección viral, acelera la degradación de HDL y de lipoproteínas de baja densidad (LDL) en el contexto de infección por SARS-CoV- ${ }^{25}$, lo que conduce a la acumulación de moléculas de lipoproteínas de muy baja densidad (VLDL) y de triglicéridos ${ }^{22}$.

Por otro lado, durante el proceso inflamatorio, la LDL y su principal lipoproteína la ApoB se oxidan en LDL oxidada, mediante la vía de receptores de lectina tipo $\mathrm{C}^{22,23}$, lo que disminuye los niveles de LDL. Se ha postulado que esto contribuye al aumento de la permeabilidad vascular causado por la infección por SARS-CoV-2 y favorece la fuga de LDL en los espacios alveolares para formar exudado, el cual contiene gran cantidad de proteínas y colesterol ${ }^{25,26}$.

El estímulo continuo de la cascada inflamatoria genera una disminución de la esterificación del colesterol, con disminución del retorno de los ésteres de colesterol al hígado, ya sea directamente por la interacción con el receptor eliminador hepático de clase $B$ tipo 1 o indirectamente después de la transferencia a LDL por la proteína de transferencia de éster de colesterol y la inclusión por los receptores de LDL hepáticos ${ }^{22}$.

Adicionalmente, el daño hepático generado por la infección por SARS-CoV-2 podría interferir con el consumo de LDL, lo cual reduciría la biosíntesis de este y la disminución de ApoA-I en la molécula de HDL. El aumento de citocinas proinflamatorias, como el TNF- $\alpha$, la IL-6 y la IL-1b, en la infección por este virus puede alterar la función hepática, afectando el transporte y la regulación del colesterol, lo que podría estar reflejado por la elevación de las transaminasas descrita en esta población ${ }^{23,25-28}$.

$\mathrm{Hu}$, et al. ${ }^{29}$ describieron que en personas con neumonía por SARS-CoV-2 ocurre un descenso del colesterol total, del colesterol HDL y del colesterol LDL hasta el noveno día de instauración de los síntomas, y que posteriormente aumentan hasta el día 16 en los sujetos del estudio que egresaban del hospital29.

La infección por SARS-CoV-2 genera una inflamación sistémica con el consecuente consumo de lipoproteínas. Esta relación entre inflamación y lipoproteínas fue descrita por $\mathrm{Li}$, et al. ${ }^{23}$, quienes documentaron que las relaciones PCR/HDL y PCR/ ApoAl están asociadas con la gravedad de la enfermedad evaluada mediante el índice SOFA (R: 0.519 y 0.516 ; $p<0.001$ ). Wei, et al. ${ }^{26}$ encontraron una correlación inversa entre las cifras de PCR y las de LDL, colesterol total y HDL (R: $-0.290,-0.332$ y -0.0351 ; $p<0.001$ ), y una relación inversa entre la IL-6, el colesterol LDL y el colesterol total (R: -0176 y P: 0.032; $R: 0,170$ y $P: 0.038$ ), siendo un determinante de gravedad en este grupo de sujetos.

\section{Conclusiones}

La infección por SARS-CoV-2 ha generado innumerables investigaciones y publicaciones, y hasta el momento no terminamos de conocer todas las manifestaciones del virus en el organismo. Estamos a la espera de una amplia vacunación, pero mientras terminan los estudios, el número de casos y de muertes sigue en aumento, y por lo tanto hay que recomendar y acatar el distanciamiento social, el adecuado uso 
del tapabocas y el lavado de manos como las estrategias fundamentales. Es importante mencionar que el Ministerio de Salud colombiano, en su plan de priorización de la población a vacunar, en la fase 1 incluirá pacientes con comorbilidad, y dentro de esta se han seleccionado las enfermedades hipertensivas, la diabetes mellitus, la enfermedad renal crónica, la enfermedad pulmonar obstructiva crónica, el asma, la infección por $\mathrm{VIH}$, el cáncer, la tuberculosis y la obesidad.

En esta revisión hemos analizado las principales manifestaciones en el metabolismo, incluyendo el sobrepeso, la obesidad, la diabetes mellitus y la dislipidemia como factores de riesgo importantes para la morbilidad y la mortalidad de las personas con COVID-19.

\section{Conflicto de intereses}

Los autores declaran que no tienen ningún conflicto de intereses en relación con esta investigación.

\section{Financiamiento}

Los autores declaran que no hubo financiamiento.

\section{Responsabilidades éticas}

Protección de personas y animales. Los autores declaran que para esta investigación no se han realizado experimentos en seres humanos ni en animales.

Confidencialidad de los datos. Los autores declaran que han seguido los protocolos de su centro de trabajo sobre la publicación de datos de pacientes.

Derecho a la privacidad y consentimiento informado. Los autores declaran que en este artículo no aparecen datos de pacientes.

\section{Bibliografía}

1. Coperchini F, Chiovato L, Croce L, Magri F, Rotondi M. The cytokine storm in COVID-19: an overview of the involvement of the chemokine/ chemokine-receptor system. Cytokine Growth Factor Rev. 2020;53:25-32.

2. Korakas E, Ikonomidis I, Kousathana F, Balampanis K, Kountouri A, Raptis A, et al. Obesity and COVID-19: immune and metabolic derangement as a possible link to adverse clinical outcomes. Am J Physiol Endocrinol Metab. 2020;319:E105-9.

3. De Lucena TMC, Da Silva Santos AF, De Lima BR, De Albuquerque Borborema ME, De Azevêdo Silva J. Mechanism of inflammatory response in associated comorbidities in COVID-19. Diabetes Metab Syndr. 2020;14:597-600

4. Ni W, Yang X, Yang D, Bao J, Li R, Xiao Y, et al. Role of angiotensin-converting enzyme 2 (ACE2) in COVID-19. Crit Care. 2020;24:422.
5. Li MY, Li L, Zhang Y, Wang XS. Expression of the SARS-CoV-2 cell receptor gene ACE2 in a wide variety of human tissues. Infect Dis Poverty. 2020;9:45.

6. Marazuela M, Giustina A, Puig-Domingo M. Endocrine and metabolic aspects of the COVID-19 pandemic. Rev Endocr Metab Disord. 2020;21:495-507.

7. Ayres JS. A metabolic handbook for the COVID-19 pandemic. Nat Metab. 2020:2:572-85.

8. Petrilli CM, Jones SA, Yang J, Rajagopalan H, Donnell LF, Chernyak Y, et al. Factors associated with hospitalization and critical illness among 4,103 patients with COVID-19 disease in New York City. medRxiv. 2020:2020.04.08.20057794.

9. Palaiodimos L, Kokkinidis DG, Li W, Karamanis D, Ognibene J, Arora S, et al. Severe obesity is associated with higher in-hospital mortality in a cohort of patients with COVID-19 in the Bronx, New York. Metab Clin Exper. 2020;108:154262.

10. Hussain A, Mahawar K, Xia Z, Yang W, El-Hasani S. Obesity and mortality of COVID-19. Meta-analysis. Obes Res Clin Pract. 2020;14:295-300.

11. Petersen A, Bressem K, Albrecht J, Thieß H-M, Vahldiek J, Hamm B, et al. The role of visceral adiposity in the severity of COVID-19: highlights from a unicenter cross-sectional pilot study in Germany. Metabolism. 2020;110:154317.

12. Bornstein SR, Dalan R, Hopkins D, Mingrone G, Boehm BO. Endocrine and metabolic link to coronavirus infection. Nature Rev Endocrinol. 2020;16:297-8.

13. Pérez-Martínez P, Carrasco Sánchez FJ, Carretero Gómez J, Gómez-Huelgas R. Resolviendo una de las piezas del puzle: COVID-19 y diabetes tipo 2. Rev Clin Esp (Barc). 2020;220:507-10.

14. Apicella M, Campopiano M, Mantuano M, Mazoni L, Copelli A, Del Prato S. COVID - 19 in people with diabetes: understanding the reasons for worse outcomes. Lancet Diabetes Endocrinol. 2020:8;782-92.

15. Kumar A, Arora A, Sharma P, Anikhindi SA, Bansal N, Singla V, et al. Is diabetes mellitus associated with mortality and severity of COVID-19? A meta-analysis. Diabetes Metab Syndr. 2020;14:535-45.

16. Mantovani A, Byrne CD, Zheng MH, Targher $\mathrm{G}$. Diabetes as a risk factor for greater COVID-19 severity and in-hospital death: a meta-analysis of observational studies. Nutr Metab Cardiovasc Dis. 2020;30:1236-48.

17. Huang I, Lim MA, Pranata R. Diabetes mellitus is associated with increased mortality and severity of disease in COVID-19 pneumonia - a systematic review, meta-analysis, and meta-regression. Diabetes Metab Syndr. 2020;14:395-403.

18. Cariou B, Hadjadj S, Wargny M, Pichelin M, Al-Salameh A, Allix I, et al. Phenotypic characteristics and prognosis of inpatients with COVID-19 and diabetes: the CORONADO study. Diabetologia. 2020;63:1500-15.

19. Wang Z, Du Z, Zhu F. Glycosylated hemoglobin is associated with systemic inflammation, hypercoagulability, and prognosis of COVID-19 patients. Diabetes Res Clin Pract. 2020;164:108214.

20. Pal R, Bhadada SK. COVID-19 and diabetes mellitus: an unholy interaction of two pandemics. Diabetes Metab Syndr. 2020;14:513-7.

21. Gupta R, Hussain A, Misra A. Diabetes and COVID-19: evidence, current status and unanswered research questions. Eur J Clin Nutr. 2020;74:864-70.

22. Sorokin A, Karathanasis S, Yang Z-H, Freeman L, Kotani K, Remaley A. COVID 19 -associated dyslipidemia: implications for mechanism of impaired resolution and novel therapeutic approaches. FASEB J. 2020;34:9843-53.

23. Li Y, Zhang Y, Lu R, Dai M, Shen M, Zhang J, et al. Changes in lipid metabolism in patients with severe COVID-19. Clin Chim Acta. 2021;517:66-73.

24. Feingold KR, Krauss RM, Pang M, Doerrler W, Jensen P, Grunfeld C The hypertriglyceridemia of acquired immunodeficiency syndrome is associated with an increased prevalence of low density lipoprotein subclass pattern B. J Clin Endocrinol Metab. 1993;76:1423-7.

25. Cao X, Yin R, Albrecht H, Fan D, Tan W. Cholesterol: a new game player accelerating vasculopathy caused by SARS-CoV-2? Am J Physiol Endocrinol Metab. 2020;319:E197-E202.

26. Wei X, Zeng W, Su J, Wan H, Yu X, Cao X, et al. Hypolipidemia is associated with the severity of COVID-19. J Clin Lipidol. 2020;14:297-304.

27. Zhang XJ, Qin JJ, Cheng X, Shen L, Zhao YC, Yuan Y, et al. In-hospital use of statins is associated with a reduced risk of mortality among individuals with COVID-19. Cell Metab. 2020;32:176-87 e4

28. Ma L, Xie W, Li D, Shi L, Mao Y, Xiong Y, et al. Effect of SARS-CoV-2 infection upon male gonadal function: a single center-based study. 2020. Disponible en: https://www.medrxiv.org/content/10.1101/2020.03.21.20037267v2

29. Hu X, Chen D, Wu L, He G, Ye W. Low serum cholesterol level among patients with COVID-19 infection in Wenzhou, China. SSRN Electronic Journal. 2020 